\title{
Elastic deformations in field-cooled vortex lattices in $\mathrm{NbSe}_{2}$
}

\author{
M. Marchevsky, A. Keurentjes, J. Aarts, and P. H. Kes \\ Kamerlingh Onnes Laboratory, Leiden University, 2300 RA Leiden, The Netherlands
}

(Received 8 August 1997)

\begin{abstract}
Simultaneous magnetic decoration of the two sides of a field-cooled (FC) superconducting sample can provide information about elasticity of the vortex lattice. We report such a decoration experiment done on a single crystal of $\mathrm{NbSe}_{2}$ and analyze the behavior of the displacement correlator $\left\langle u^{2}(r, z)\right\rangle$ for the FC lattice. The ratio of the elastic constants $c_{66} / c_{44}$ is found to be strongly renormalized downward, probably by the presence of topological defects. [S0163-1829(98)00409-3]
\end{abstract}

\section{INTRODUCTION}

Vortices in superconductors are often modeled as rods that pass straight through a sample. Realistic models however take into account that vortex lines are flexible and can bend in the material. Interplay between three-dimensional (3D) elastic and plastic distortions due to pinning and thermal fluctuations results in a rich physical picture of the vortex lattice that involves the phenomena of collective pinning, melting, and (in the case of strong anisotropy) decoupling transitions. To study these phenomena in more detail it is of importance to probe the development of positional fluctuations in the vortex lattice not only in the transverse direction (normal to the field) but also in the longitudinal direction. Although indirect measurements have already proven that the vortex lattice can behave as a system with 3D disorder, direct information on the vortex displacement in longitudinal direction was until recently only obtainable from the results of neutron scattering or muon spin rotation. Such experiments were done on both conventional ${ }^{1-3}$ and high- $T_{c}$ materials, ${ }^{4-6}$ and in the latter case provided evidence on fluxlattice melting and decoupling transitions. Neutron experiments can only be performed on large and homogeneous samples and their scope is limited to the field range $H_{c 1}$ $<H \ll H_{c 2}$. Another complication is that in platelet samples vortex bending due to geometrical effects affects the scattering intensity and should be properly taken into account.

Normally, magnetic decoration gives information on the distribution of the vortex lines as they emerge at the sample surface. The point was raised by Huse, ${ }^{7}$ whether the patterns obtained in magnetic decoration experiments are predominantly determined by the flux distribution in the bulk or just by surface effects. It was also argued, ${ }^{8}$ that surface pinning effects can be distinguished from "bulk' effects by studying the behavior of the vortex lattice structure factor at small wave vectors. Recently a novel type of decoration experiment, introduced in the work of Yao et al., ${ }^{9}$ and later of Yoon et al., ${ }^{10}$ has clarified some of these problems. The authors decorated a crystal of the high- $T_{c}$ superconductor Bi$\mathrm{Sr}-\mathrm{Ca}-\mathrm{Cu}-\mathrm{O}$ simultaneously at both sides and found that it is possible to map the two vortex patterns onto each other. In this way not only the in-plane distribution of the vortex lines but also their wandering inside the sample was investigated. It was shown both that the vortices in $\mathrm{Bi}-\mathrm{Sr}-\mathrm{Ca}-\mathrm{Cu}-\mathrm{O}$ at low fields behave as lines and that the vortex decoration patterns are a result of bulk disorder and thermal fluctuations, not of surface disorder.

Below, we show the results of double-sided decoration of a field-cooled vortex lattice in $\mathrm{NbSe}_{2}$, and we discuss how to obtain information on the dimensionality of the structure and on the ratio of the elastic constants for tilt and shear deformation. For this, we follow a different approach than used in Refs. 9,10. The analysis presented there was based on the long-wavelength behavior of the vortex structure function, and the assumption that this can be described in terms of a flux-line liquid as discussed by Marchetti and Nelson. ${ }^{8}$ Rather, we will study the behavior of the displacement of the vortices with respect to their ideal lattice positions, which is the starting point for collective-pinning theory. The connection between the vortex displacements on both sides of the sample and the elastic constants of the lattice will be discussed in Sec. II. In Sec. III we present the decoration results, and we discuss the important problem of determining the temperature at which the vortex lattice freezes in the structure visualized by the decoration (the so-called quenching temperature). In Sec. IV the results are analyzed quantitatively, leading to an estimate for the ratio between the shear modulus and the tilt modulus of the vortex lattice at the quenching temperature.

\section{THE BEHAVIOR OF THE DISPLACEMENT CORRELATOR IN DOUBLE-SIDED DECORATION}

Consider a dislocation-free vortex lattice in a random disorder potential. It was shown by Larkin ${ }^{11}$ and Ovchinnikov (LO) that such a potential destroys long-range translational order in the vortex lattice. Short-range order, however, still persists in domains defined by a transverse correlation length $R_{c}$ perpendicular to the applied field, and a longitudinal correlation length $L_{c}$ parallel to the field. These characteristic lengths can be extracted from the behavior of the displacement correlator $\left\langle u^{2}(\mathbf{r}, z)\right\rangle \equiv\left\langle[\mathbf{u}(\mathbf{r}, z)-\mathbf{u}(\mathbf{0}, 0)]^{2}\right\rangle$ (see Fig. 1). Here $\mathbf{u}(\mathbf{r}, z)$ and $\mathbf{u}(\mathbf{0}, 0)$ denote the $2 \mathrm{D}$ displacement vectors of the actual vortex positions with respect to the ideal lattice positions separated by a vector $\mathbf{r}$ in a plane perpendicular to the applied magnetic field. The average should be taken over all possible configurations of the random point disorder. Correlated regions are elastically independent of each other and their dimensions $R_{c}$ and $L_{c}$ are defined by the conditions 


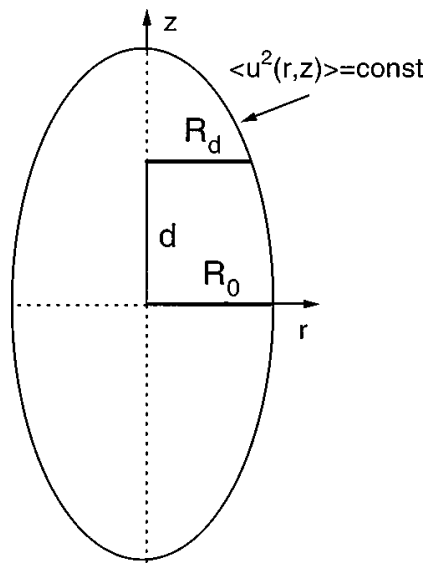

FIG. 1. A sketch of the ellipsoidal surface of constant amplitude of the displacement correlator $\left\langle u^{2}(r, z)\right\rangle=$ const. Cross sections of the ellipsoid at $z=0$ and $z=d$ can be probed with the results of the double-sided decoration.

$$
\left\langle u^{2}\left(R_{c}, 0\right)\right\rangle=\left\langle u^{2}\left(0, L_{c}\right)\right\rangle=r_{p}^{2},
$$

where $r_{p}$ denotes the range of the elementary pinning interaction. It can be taken equal to the vortex core diameter (e.g., $r_{p} \simeq \xi$ ) for fields $B \lesssim 0.25 B_{c 2}$.

The displacement correlator can be calculated from the decoration pattern to a good approximation. To do so, first a perfect vortex lattice with a lattice parameter equal to that of the real pattern is generated and fit to the real lattice by minimization of the root-mean-square (rms) displacement between the two patterns. Next, the correlator $\left\langle u^{2}(r)\right\rangle$ is computed for every lattice point and the average is taken over the entire pattern. Strictly speaking, averaging over the pattern is not the same as averaging over all possible configurations of the random disorder, as assumed in the LO theory. However, if the dimensions of the pattern are (much) larger than $R_{c}$, the result of the direct calculation can be taken as an approximation. It should be pointed out that, in the experiment, the displacement correlator found from the decoration pattern is usually scaled to the flux-lattice constant $a_{0}$, which is the only natural length scale available. An experimentally relevant length scale is therefore set by the conditions

$$
\left\langle u^{2}\left(L_{a}\right)\right\rangle=\left\langle u^{2}\left(R_{a}\right)\right\rangle=a_{0}^{2},
$$

where $L_{a}$ and $R_{a}$ are called the longitudinal and transverse lattice correlation lengths. Generally for a vortex lattice the inequalities $L_{a} \gg L_{c}$ and $R_{a} \gg R_{c}$ hold. It is important to emphasize here the different meaning of the pinning $\left(R_{c}, L_{c}\right)$ and lattice $\left(R_{a}, L_{a}\right)$ characteristic length scales. It is the lattice correlation length, either transverse or longitudinal, which is measured by different visualization methods, rather than the pinning correlation lengths $R_{c}$ and $L_{c}{ }^{12}$ In a realistic vortex lattice, as seen in field-cooled FC decoration experiments, plastic deformations and topological defects are almost always present. In that case, one cannot use the LO theory directly to determine pinning strength and elastic constants from the overall pictures. In order to analyze the data in a quantitative way, topological defects have to be avoided as far as possible. We will come back to this point in the analysis.
A double-sided decoration experiment yields two displacement correlators, $\left\langle u^{2}(r, 0)\right\rangle$ and $\left\langle u^{2}(r, d)\right\rangle$, with $d$ being the sample thickness. In order to understand how to extract the information on the elastic constants, consider 3D surfaces of constant displacement amplitude $\left\langle u^{2}(R, L)\right\rangle=$ const. These surfaces are cigar-shaped, with the axis $R$ in the transverse direction and axis $L$ in the longitudinal one (see Fig. $1)$. The ratio of the axes is given by the competition between tilt and shear energy densities $c_{44}(u / L)^{2}$ and $c_{66}(u / R)^{2}$, which leads to the simple expression ${ }^{13}$

$$
L \simeq R \sqrt{\frac{c_{44}\left(k_{\perp}, k_{z}\right)}{c_{66}}},
$$

where $c_{66}$ is the shear modulus of the vortex lattice, and $c_{44}$ the tilt modulus. Now assume that the "cigar" has the profile of an ellipsoid. Its equation is then

$$
\left(\frac{r}{R}\right)^{2}+\left(\frac{z}{L}\right)^{2}=1
$$

Substituting expression (3) for $L$, Eq. (4) can be rewritten as

$$
r^{2}+z^{2} \frac{c_{66}}{c_{44}\left(k_{\perp}, k_{z}\right)}=R^{2} .
$$

As a next step, for any displacement value $u_{0}$ the transverse axis $R_{0}$ can be found via the in-plane correlator $\left\langle u^{2}(r, 0)\right\rangle$ by using the condition $\left\langle u^{2}\left(R_{0}, 0\right)\right\rangle=u_{0}^{2}$. Then the condition, $\left\langle u^{2}\left(R_{d}, d\right)\right\rangle=u_{0}^{2}$ allows us to find the cross section of the ellipsoid $R_{d}$ at $z=d$ (see Fig. 1). By substituting $R_{0}$ and $R_{d}$ in Eq. (5), the ratio of elastic constants $c_{66} / c_{44}\left(k_{\perp}, k_{z}\right)$ can be determined. This experimental number then can be compared to the number calculated from theoretical expressions for $c_{66}$ and $c_{44}$. Note that for such a comparison it will be necessary to know the quenching temperature $T_{q}$ of the lattice, since the elastic constants depend on the temperature. In the next section, we discuss how to determine $T_{q}$ experimentally.

\section{EXPERIMENTS: QUENCHING TEMPERATURE AND DOUBLE-SIDED DECORATION}

In a field-cooled (FC) experiment a pinning force sets in at some quenching temperature $T_{q}<T_{c}$ and the vortex configuration in the sample "freezes" at this temperature. Therefore the pattern observed in such experiments essentially represents the vortex configuration quenched at $T_{q}$ which can be substantially higher than the actual temperature of the decoration. To analyze the FC vortex structure in terms of elasticity and pinning, the knowledge of the quenching temperature is essential. We have done an experiment to determine this temperature for $\mathrm{NbSe}_{2}$.

Due to the demagnetization effect in a platelet-shaped superconducting sample, the equilibrium magnetic induction in the middle is only slightly different from the applied field. This can be used in the following way. During the cooldown the applied field $H_{a}$ was controlled by and changed linearly with the actual temperature of the sample. As a result of this procedure, the induction $B_{m}$ in the middle of the sample at low temperature is just the one that was frozen in at the quenching temperature $T_{q}$, because at $T_{q}$ the pinning poten- 
tial rises steeply so that the vortices are trapped. In addition, the shielding current in the vicinity of the sample center is close to zero. Since the actual dependence between the external field and temperature is known from the calibration curve of $H_{a}(T)$, one can estimate $T_{q}$ by measuring $B_{m}$, either by decoration or by a miniature Hall sensor. The details of this experiment are given elsewhere. ${ }^{14}$ We found that for $\mathrm{NbSe}_{2}$ the quenching temperature $T_{q}$ is high, just about 100 $\mathrm{mK}$ below $T_{c}\left(T_{c}=7.1 \mathrm{~K}\right)$.

The double-sided decoration experiment was performed on a single crystal of $\mathrm{NbSe}_{2}$ of about $1 \mathrm{~mm}$ in diameter. It was cleaved at both sides prior to decoration and glued with one side to a copper holder. The final thickness of the cleaved crystal was determined by electron microscopy to be about $12.5 \mu \mathrm{m}$. A tiny slit in the edge of the holder plate allowed a small area $\left(\sim 0,05 \mathrm{~mm}^{2}\right)$ on both crystal sides to be exposed to the magnetic particles in the course of the decoration procedure. Except for this specialized sample holder, a conventional arrangement was used in the experiment. The sample was cooled in an external field of $5.0 \mathrm{mT}$ applied along the $c$ axis of the crystal, and decorated at $T_{\mathrm{dec}}=1.4 \mathrm{~K}$. Heating of the sample during decoration was moderate with temperature rising to about $2.9 \mathrm{~K}$. Electron microscope images of the decoration patterns were obtained from both sides of the crystal, from which the positions of the vortices were determined and Delauney triangulation was performed. The resulting triangulated patterns are shown in Fig. 2. The presence of a crystal edge in both original photographs served as a natural reference in the initial attempt to match the patterns. However, the final accurate matching was carried out by using an algorithm, ${ }^{15}$ in which the rms displacement of the vortex centers as they cross the crystal was sequentially minimized with respect to the aspect ratio of the in-plane axes, relative rotation angle and translation vector.

The following procedure was used. First the best rotation angle and aspect ratio were found. Minimization with respect to the aspect ratio of the images was necessary due to the existing inaccuracy of the digital frame grabber of the SEM. For every value of the aspect ratio $A(0.98 \leqslant A \leqslant 1.02$, with steps of 0.001$)$, a rotation was performed over an angle $\varphi$, leading to a displacement vector $\mathbf{r}(A, \varphi)$ which was added to the coordinates of the pixels in one of the patterns. Then the rms displacement $\Delta$ was calculated. In this way, the minimum $\Delta\left(A_{\text {best }}, \varphi_{\text {best }}\right)$ was found. Finally $\Delta\left(A_{\text {best }}, \varphi_{\text {best }}, \delta \mathbf{r}\right)$ was minimized with respect to a relative translation $\delta \mathbf{r}$ $=|\mathbf{r}(L)-\mathbf{r}(0)|$ between the top and bottom patterns.

The best match of the two patterns is shown in Fig. 3 . There are 1644 points in each of the matching parts of the patterns. One sees from the picture that positional shifts of the corresponding points are small and that all the topological defects at both sides match very well. The rms displacement of the flux-line positions $\left\langle|\mathbf{r}(L)-\mathbf{r}(0)|^{2}\right\rangle^{1 / 2}$ calculated after all minimizations was found to be about $17 \%$ of the lattice parameter $a_{0}$. In the paper of Yao et al. ${ }^{9}$ a similar number was found for a $\mathrm{Bi}-\mathrm{Sr}-\mathrm{Ca}-\mathrm{Cu}-\mathrm{O}$ sample with a thickness of $10 \mu \mathrm{m}$ cooled in a field of $1.2 \mathrm{mT}$. The authors interpreted this as a measure of the wandering of the vortex lines as they pass through the crystal. We think, however, that this number should be considered with caution. In fact, the inaccuracy in finding the vortex centers from the decoration pattern is normally about $5-10 \%$ of $a_{0}$. This substan-

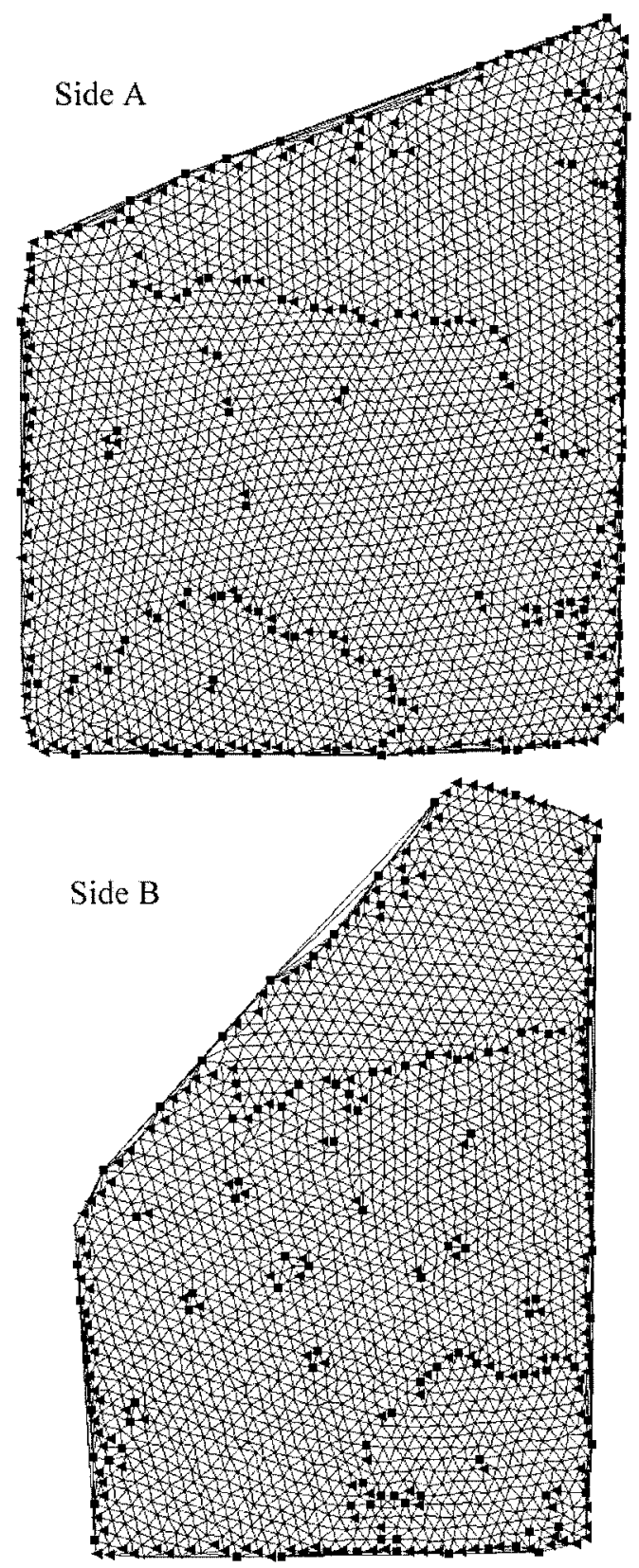

FIG. 2. Delauney triangulations of the vortex patterns at the top (a) and bottom (b, mirrored) side of the crystal. Boundaries of the VL grains consisting of alternating fivefold- $(\boldsymbol{\Delta})$ and sevenfold-

(ם) coordinated lattice sites are visible.

tially contributes to the observed value of the rms displacement, see below.

We also note that in our case, during the cooldown from $T_{q}$ some additional shifts of vortices and probably even topological defect formation can occur. The displacement of the individual lines at $T_{\text {dec }}$ from their positions at $T_{q}$ can be of the order of $\xi\left(T_{q}\right)$. We estimate $\xi\left(T_{q}\right)$ at $70 \mathrm{~nm}$, which is significant compared to $c_{L} a_{0}\left(a_{0} \simeq 0.69 \mu \mathrm{m}\right)$, with $c_{L}$ the Lindemann constant of order 0.2 .

\section{ANALYSIS AND DISCUSSION}

First of all, Fig. 3 clearly shows that the displacement of the individual lines across the sample is small in comparison 


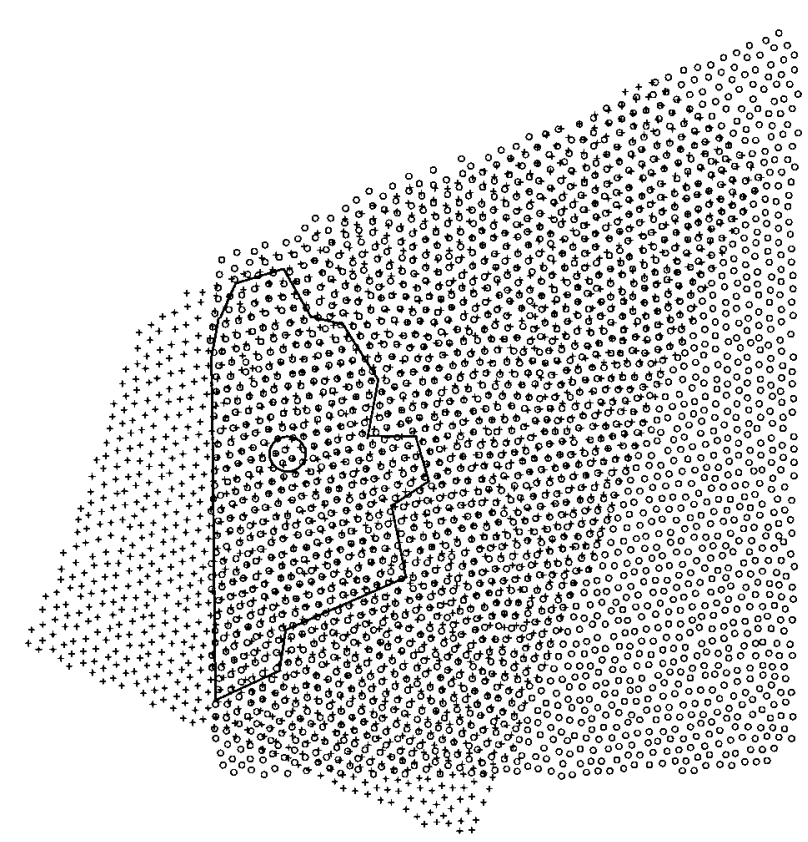

FIG. 3. Result of double-sided decoration on $\mathrm{NbSe}_{2}$ at $5.0 \mathrm{mT}$. The best match of the two patterns obtained after sequential minimization of the rms displacement $\left\langle|\mathbf{r}(\mathrm{L})-\mathbf{r}(0)|^{2}\right\rangle^{1 / 2}$ with respect to rotation and translation is shown. The resulting minimal rms displacement is approximately 0.165 of the lattice parameter indicating long-range order along the field direction. The outlined part of the lattice (excluding the encircled part, containing an interstitial) without defects was used for the numerical analysis.

with $a_{0}$. In other words, the longitudinal lattice correlation length $L_{a}$ is much larger than the sample thickness $d$ and no traces of vortex entanglement are seen. Structurally the vortex system looks very 2D-like. In the transverse plane, however, the translational order is poor. It is destroyed by the grain boundaries and also by several edge dislocations inside the grains. In order to estimate the transverse displacement of the lattice points, the displacement correlator $\left\langle u^{2}(r)\right\rangle$ $=\left\langle[\mathbf{u}(\mathbf{r}, z)-\mathbf{u}(\mathbf{0}, 0)]^{2}\right\rangle$ was calculated for a part of the lattice free of topological defects, taken within the middle vortex grain (see Figs. 2 and 3). First, a reference lattice was generated, and fitted to the real pattern at one side with the same procedure as described above for matching "top" and "bottom" patterns to each other. Next, the displacements of points at both sides with respect to the reference lattice were determined. Finally, two correlators were calculated: $\left\langle u^{2}(r, 0)\right\rangle$, the usual in-plane correlator for one of the patterns and $\left\langle u^{2}(r, d)\right\rangle$, a correlation across the sample thickness $d$, accounting for the growth of the vortex displacements along both transverse and longitudinal directions. The results of the calculations are shown in Fig. 4. It is seen that both correlators differ for the shortest distances $\sim 1-2 a_{0}$, while for $r \gtrsim 4-5 a_{0}$ they merge.

Let us first discuss the in-plane result $\left\langle u^{2}(r, 0)\right\rangle$. If this function is linearly extrapolated to $r=0$, the constant at the origin denotes the magnitude of the noncorrelated displacement. Two major factors contribute to this displacement. One of them is the measuring error in the determination of the vortex positions, as we already mentioned. Another contribution may come from the noncorrelated short- $\left(\sim a_{0}\right)$ wavelength deformations caused by thermal fluctuations or

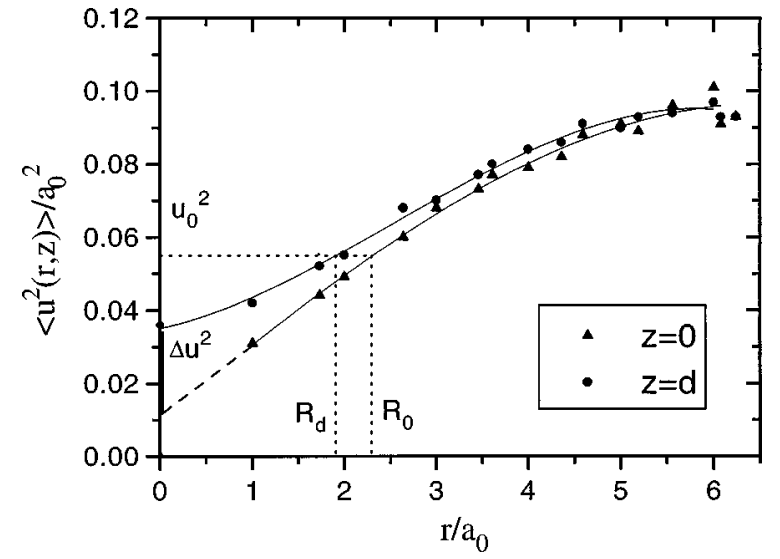

FIG. 4. Displacement correlation functions $\left\langle[\mathbf{u}(\mathbf{r}, \mathrm{z})-\mathbf{u}(\mathbf{0}, 0)]^{2}\right\rangle$ calculated for a defect-free piece (349 points) of the vortex grain seen in the middle of the pattern in Fig. 2, in-plane $(z=0)$ and across the sample thickness $(z=d)$. The lines are guides for the eye. $\Delta u^{2}$ signifies the longitudinal wandering of the vortex lines across the sample thickness $d$; the values of $u_{0}^{2}, R_{0}$, and $R_{d}$ used in estimating the ratio $c_{66} / c_{44}$ are also indicated.

pinning. As a result of such deformations, the tips of the vortex lines at the surface will be randomly displaced. One should note here that both these factors are expected to give roughly the same $r$-independent contribution in the displacement correlator $\left\langle u^{2}(r \rightarrow 0, z)\right\rangle$ for $z=0$ and for $z=d$. At short length scales $\left(r \gtrsim a_{0}\right)$ the displacement correlator grows roughly linearly with distance. Looking at the numbers, and using $a_{0} \simeq 0.69 \mu \mathrm{m}$ and $\xi\left(T_{q}\right) \simeq 70 \mathrm{~nm}$, we see that $\left\langle u^{2}\right\rangle$ is already comparable to $\xi^{2}$ at $r \simeq a_{0}$, meaning that $R_{c}$ is roughly equal to $a_{0}$. For the correlator $\left\langle u^{2}(r, d)\right\rangle$, we find that the noncorrelated displacement at $r=0$ is increased compared to its in-plane magnitude. The difference at $r=0$, $\Delta u^{2} \simeq\left(0.15 a_{0}\right)^{2}$, results entirely from the longitudinal wandering of the vortex lines across the sample thickness $d$, because all other contributions are the same.

Let us now calculate the ratio of the elastic constants as outlined in Sec. II. We choose an arbitrary constant displacement $u_{0}$ of about $0.2 a_{0}$, where the tranverse distances are $R_{0} \simeq 2.3 a_{0}$ and $R_{d} \simeq 1.9 a_{0}$ (as indicated in Fig. 4). Using Eq. (5) and $d=12.5 \mu \mathrm{m}$, we find $c_{66} / c_{44} \simeq 5 \times 10^{-3}$. To put this number in perspective, we next consider a theoretical estimate. The dispersive tilt modulus $c_{44}(\mathbf{k})$ consists of a bulk term $c_{44}^{\text {bulk }}(\mathbf{k})$ and a single vortex contribution $c_{44}^{s v}\left(k_{z}\right)$ :

$$
c_{44}(\mathbf{k})=c_{44}^{\text {bulk }}(\mathbf{k})+c_{44}^{s v}\left(k_{z}\right)
$$

where $^{16}$

$$
\begin{aligned}
c_{44}^{s v}= & \frac{B^{2}}{2 \mu_{0} \lambda^{2} K_{\mathrm{BZ}}^{2}}\left\{\varepsilon^{2} \ln \left(\frac{(\lambda / \varepsilon \xi)^{2}}{1+\left(\lambda^{2} / \varepsilon^{2}\right) K_{\mathrm{BZ}}^{2}+\lambda^{2} k_{z}^{2}}\right)\right. \\
& \left.+\frac{1}{\lambda^{2} k_{z}^{2}} \ln \left(1+\frac{\lambda^{2} k_{z}^{2}}{1+\lambda^{2} K_{\mathrm{BZ}}^{2}}\right)\right\}
\end{aligned}
$$

and

$$
c_{44}^{\text {bulk }}=\frac{B^{2}}{\mu_{0}} \frac{1}{1+\left(\lambda^{2} / \varepsilon^{2}\right) k_{\perp}^{2}+\lambda^{2} k_{z}^{2}} .
$$


The shear modulus $c_{66}$ is given by the standard expression

$$
c_{66}=\frac{\Phi_{0} B}{16 \pi \mu_{0} \lambda^{2}} \quad\left(\lambda>a_{0}, B \ll B_{c 2}\right) .
$$

In the above expressions, $k_{\perp}$ and $k_{z}$ are the wave vectors of the deformation perpendicular and along the vortex lines, respectively, $\Phi_{0}$ is the flux quantum, $K_{\mathrm{BZ}}=\left(4 \pi B / \Phi_{0}\right)^{1 / 2}$ is the Brillouin-zone boundary, and $\varepsilon$ is the anisotropy parameter. When the ratio $c_{66} / c_{44}$ ratio is estimated using Eqs. (6)-(9) with wave vectors $k_{\perp} \simeq \pi /\left(2 a_{0}\right)$ and $k_{z}=\pi / d$ corresponding to the relevant volume of the ellipsoid and taking $\lambda=\lambda\left(T_{q}\right)$, a value $3.8 \times 10^{-1}$ is obtained. Note that the estimate is almost independent of temperature close to $T_{c}$. The result is two orders of magnitude larger than the experimental value, showing that the vortices are extremely straight.

Our results can be compared with the results of Refs. 9,10 obtained on Bi-Sr-Ca-Cu-O. It is rather surprising that despite the differences in the superconducting properties of the two materials one sees very similar bulk behavior of the vortex lines. The ratio of $c_{66} / c_{44}$ determined for Bi-Sr-Ca$\mathrm{Cu}-\mathrm{O}$ from the double-sided decoration data ${ }^{10}$ is also much smaller ( $\sim 200$ times) than that calculated by the authors. In our experiment, one possible reason for the discrepancy between the measured and theoretical value of the ratio of elastic constants might be the influence of the deformation field from the dislocations and defects which surround the analyzed part of the lattice. When the dimensions of the defectfree part are small $\left(\sim 10-15 a_{0}\right)$, the internal deformations (still being elastic) are coupled with the peripheral plastic distortions. The plastic deformations produce transverse lattice displacements of the order of $a_{0}$ at short length scales $r \gtrsim a_{0}$. Our experiment clearly shows that in a field-cooled lattice, those displacements are not transferred to the longitudinal ones according to the relation of Eq. (3). On the contrary, the grain boundaries or disclination rows go straight through the thick $\left(d \gg a_{0}\right)$ crystal, resulting in a considerable change in the anisotropy of the elastic distortions of the near lattice parts. This is consistent with recent theoretical results. ${ }^{17}$ Also, a downward renormalization of the shear modulus at distances larger than the distance between dislo- cations (or grain boundaries) should be expected. ${ }^{18}$ Our result suggests therefore a much larger number for the $L / R$ ratio in the vortex lattice in presence of plastic deformations. In the patterns studied in Refs. 9,10 plastic lattice defects were also present within the analyzed part of the patterns, which might have affected the result even stronger.

Recently an interesting suggestion was made in Ref. 19, where the phenomena of melting (or freezing) and depinning (or quenching) of the vortex lattice were connected to each other. According to this model the average distance between dislocations is about equal to $R_{c}$ at the depinning (or quenching) crossover. In our case it is then suggestive to take the grain size at $T_{q}$ equal to $R_{c}$. In an earlier analysis ${ }^{20}$ of the granular vortex structure in $\mathrm{Nb}$, the vortex grain size was interpreted as being $R_{c}$ and its field dependence was found to be consistent with the predictions of the LO theory. In our experiment, defects are mostly aligned in the grain boundaries. Therefore, an alternative approach to estimate the ratio of elastic constants may be to equate the wavelength of the transverse deformations in $c_{44}(\mathbf{k})$ to the distance between the grain boundaries. If one substitutes the wave vector corresponding to the average grain size $\left[k_{\perp} \sim \pi /\left(30 a_{0}\right)\right]$ in the standard expression for $c_{44}(\mathbf{k})$, the ratio of elastic constants is $\sim 5.4 \times 10^{-3}$, i.e., much closer to the experimental result.

In conclusion, we performed a double-sided decoration experiment on $\mathrm{NbSe}_{2}$ and estimated the ratio of the shear modulus to the tilt modulus of the vortex lattice. We found this ratio to be much smaller than that predicted by the elastic theory at the quenching temperature for the vortex lattice. We think that topological defects are (partly) responsible for the observed anisotropic renormalization of the elastic displacement.

\section{ACKNOWLEDGMENTS}

We thank D. Nelson, V. Vinokur, and A. Koshelev for stimulating discussions and M. C. Miguel and M. Kardar for providing us with their recent results before publication. We are indebted to J. V. Waszczak for the $\mathrm{NbSe}_{2}$ single crystals. This work is part of the research program of the Stichting voor Fundamental Onderzoek der Materie, which is financially supported by NWO.
${ }^{1}$ J. W. Lynn, N. Rosov, T. E. Grigereit, H. Zhang, and T. W. Clinton, Phys. Rev. Lett. 72, 3413 (1994).

${ }^{2}$ U. Yaron, R. N. Kleiman, C. S. Oglesby, E. Bucher, B. Batlogg, D. J. Bishop, and K. Mortensen, Nature (London) 376, 753 (1995).

${ }^{3}$ P. L. Gammel, D. A. Huse, R. N. Kleiman, B. Batlogg, C. S. Oglesby, E. Bucher, D. J. Bishop, T. E. Mas, and K. Mortensen, Phys. Rev. Lett. 72, 278 (1994).

${ }^{4}$ E. M. Forgan, D. McK Paul, H. A. Mook, P. A. Timmins, H. Keller, S. Sutton, and J. S. Abell, Nature (London) 343, 735 (1990).

${ }^{5}$ R. Cubbit, E. M. Forgan, G. Yang, S. L. Lee, D. McK Paul, H. A. Mook, M. Yethiraj, P. H. Kes, T.-W. Li, A. A. Menovsky, and Z. Tarnavski, Nature (London) 365, 407 (1995).

${ }^{6}$ S. L. Lee, M. Warden, H. Keller, J. W. Schneider, D. Zech, P. Zimmermann, R. Cubitt, E. M. Forgan, M. T. Wylie, P. H. Kes,
T.-W. Li, A. A. Menovsky, and Z. Tarnawski, Phys. Rev. Lett. 75, 922 (1995).

${ }^{7}$ D. A. Huse, Phys. Rev. B 46, 8621 (1992).

${ }^{8}$ M. C. Marchetti and D. R. Nelson, Phys. Rev. B 47, 12214 (1993).

${ }^{9}$ Z. Yao, S. Yoon, H. Dai, S. Fan, and Ch. Lieber, Nature (London) 371, 777 (1994).

${ }^{10}$ S. Yoon, Z. Yao, H. Dai, and C. M. Lieber, Science 270, 270 (1995).

${ }^{11}$ A. I. Larkin, Zh. Éksp. Teor. Fiz. 58, 1466 (1970) [Sov. Phys. JETP 31, 784 (1970)]; A. L. Larkin and Yu. N. Ovchinnikov, J. Low Temp. 34, 409 (1979).

${ }^{12}$ This was specifically noted recently with respect to the interpretation of neutron-scattering results: T. Giamarchi and P. Le Doussal, Phys. Rev. Lett. 75, 3372 (1995).

${ }^{13}$ G. Blatter, M. V. Feigelman, V. B. Geshkenbein, A. I. Larkin, 
and V. M. Vinokur, Rev. Mod. Phys. 66, 1125 (1994).

${ }^{14}$ M. Marchevsky, J. Aarts, and P. H. Kes, Physica C 282-287, 2083 (1997).

${ }^{15}$ M. C. Marchetti and D. Nelson, Phys. Rev. B 52, 7720 (1995).

${ }^{16}$ G. Blatter, V. Geshkenbein, A. I. Larkin, and H. Nordborg, Phys. Rev. B 54, 72 (1996).
${ }^{17}$ M. C. Miguel and M. Kardar (private communication).

${ }^{18}$ R. Wördenweber, Ph.D. thesis, Leiden University, 1987.

${ }^{19}$ C. Tang, X. Ling, S. Bhattacharya, and P. M. Chaikin, Europhys. Lett. 35, 597 (1996).

${ }^{20}$ L. Ya. Vinnikov and I. V. Grigorieva, Pis'ma Zh. Eksp. Teor. Fiz. 47, 89 (1988); I. V. Grigorieva, Supercond. Sci. Technol. 7, 161 (1994). 